J. Austral. Math. Soc. 19 (Series A), (1975), 103-120.

\title{
STRONG MAXIMUM PRINCIPLES FOR WEAKLY COUPLED SYSTEMS OF QUASILINEAR PARABOLIC INEQUALITIES
}

\author{
M. A. DOW
}

(Received 19 November 1973)

Communicated by J. P. O. Silberstein

\begin{abstract}
Výborný and I (1972) proved maximum principles for a quasilinear elliptic operator where the boundary satisfied a smoothness condition weaker than the interior sphere property. In this paper I extend these to parabolic operators of a similar form and through a simple device to weakly coupled systems of such operators. Finally, I extend all of these results to an operator similar to the "parabolic" case of an operator introduced by Redheffer (1971). His conditions on the coefficients are replaced by conditions analogous to those Dow and Výborný (1972).
\end{abstract}

Výborný and I (1972) proved interior and boundary maximum principles and a consequent uniqueness theorem for a quasilinear elliptic operator. The conditions on the coefficients were similar to those used by Pucci (1957) and (1958) for linear operators and by Redheffer (1962), Výborný (1963), and Horáček and Výborný (1966) for quasilinear operators. The boundary satisfied a smoothness condition weaker than the interior sphere property.

The object of this paper is to extend these theorems to parabolic operators

$$
P u=\sum_{i, j=1}^{n-1} a_{i j}(x, u, D u) D_{i j} u+\sum_{i=1}^{n} b_{i}(x, u, D u) D_{i} u+d(x, u, D u) u+a(x, u, D u)
$$

with $b_{n}(x, u, 0)$ negative and usually bounded away from zero. Here $D_{i} u=\partial u / \partial x_{i}$ and $D u=\left(D_{1} u, \cdots, D_{n-1} u\right)$. Through a simple device, the results generalize easily to weakly coupled systems of such operators

$$
P_{\mu} u_{\mu}+\sum_{v=1}^{p} h_{\mu v}\left(x, u_{v}, D u_{v}\right) u_{v}, \mu=1, \cdots, p .
$$

Finally, I extend these results to systems in which $P_{\mu}$ is replaced by an operator similar to the "parabolic" case of an operator used by Redheffer (1971). His 
conditions on the coefficients are replaced by somewhat more general conditions similar to those of Dow and Výborný (1972).

A comprehensive discussion and bibliography to 1966 on the maximum principle for weakly coupled systems can be found in Protter and Weinberger's monograph (1967). Further maximum principles for systems have been proved by Ako (1968), Khusnytdinova (1967), Ladyzhenskaya, Solonnikov and Ural'tseva (1968), Wąsowski (1970), and Moisak (1971), and by Stroock (1970), who used stochastic methods.

\section{Definitions and basic lemma}

In what follows, $B$ denotes a continuous, positive function on $(0, \infty)$ with

$$
\int_{0}^{a} B<\infty
$$

for some $a>0$. Without loss of generality, $B$ is assumed bounded away from zero by a positive constant $B_{0}$.

The symbol $f$ denotes a non-negative function on $(0, \infty) \times[0, \infty)$ satisfying

(i) $f(t, 0)=0$ for all $t$ in $(0, \infty)$,

(ii) $f$ is non-decreasing in its second variable,

(iii) there are constants $A>0$ and $c>0$ such that for each $\varepsilon>0$ there is a supersolution $\phi_{\varepsilon}$ to the problem $\phi^{\prime}=c f(t, \phi)$ on $[0, A]$ with $0<\phi_{\varepsilon}(t) \leqq \varepsilon$ on $[0, A]$.

Here a supersolution is a function $\phi$ defined and continuous on $[0, A]$, differentiable on $(0, A)$, satisfying the inequality $\phi^{\prime}(t) \geqq \operatorname{cf}(t, \phi(t))$ on $(0, A)$. For Theorems 1.1 and 2.1, condition (ii) can be weakened slightly (see Dow and Výborný (1972), $\S 2.2)$.

Let $G$ be a bounded open set in $R^{n}$. Points in $R^{n}$ are denoted by $x=\left(x_{1}, \cdots, x_{n}\right)$. We assume $G$ is connected although this will not always be needed. Let $P$ be the parabolic operator described in the introduction with coefficients defined on $\bar{G} \times R^{1} \times R^{n-1}$. The operator $P$ is to be degenerate elliptic-parabolic relative to the function $u$ in the sense that

$$
\sum_{i, j=1}^{n-1} a_{i j}(x, u, 0) \lambda_{i} \lambda_{j} \geqq 0
$$

for all $\lambda$ in $R^{n-1}$ and all $x$ in $G$.

For each point $y$ in $\bar{G}$ we define the propagation sets $T_{y}$ and $S_{y}$. The set $T_{y}$ is the connected component of the set $G \cap\left(x_{n}=y_{n}\right)$ containing $y$ if $y \in G$ or with $y$ in its boundary if $y \in \partial G ; S_{y}$ is the set of all points in $G$ that can be connected to $y$ by a path lying in $G$, except perhaps for $y$, along which $x_{n}$ is non-decreasing from $x$ to $y$. For $y$ in $G$ these are the usual sets described by Nirenberg (1953); for $y$ in $\partial G$ the set $S_{y}$ was used by Výborny (1958). The closures of these sets relative to $G$ 
are denoted by $\hat{S}_{y}$ and $\hat{T}_{y}$; the bar notation $S_{y}$ and $\hat{T}_{y}$ is reserved for closure relative to $R^{n}$.

Suppose $y \in \partial G$ and $N$ is a neighbourhood of $y$. If the boundary of $G$ permits it, $\tau$ denotes a function on $\bar{N} \cap \bar{G}$ satisfying

(i) $\tau \in C^{1}(\bar{N} \cap \bar{G}) \cap D^{2}(\bar{N} \cap G)$,

(ii) $\tau=0$ on $\partial G \cap \bar{N}$ and $\tau>0$ on $\bar{N} \cap G$, and

(iii) $0<m \leqq|D \tau| \leqq M<\infty$ on $\bar{N} \cap G$.

In (i), $D^{2}(\bar{N} \cap G)$ is the set of twice differentiable functions on $\bar{N} \cap G$. Notice that the condition $\tau \in C^{1}(\bar{N} \cap \bar{G})$ implies the existence of an interior normal to $\partial G$ at $y$.

The operator $P$ is said to satisfy condition $Q$ relative to $u$ at $y$ in $\partial G$ if there are functions $f, B$ and $\tau$ and a neighbourhood $N$ of $y$ all satisfying the above conditions and also

(a) $|e(x, u, 0)-e(x, u, D u)| \leqq f(\tau(x),|D u(x)|)$

for $e=a_{i j}(i, j=1, \cdots, n-1), b_{i}(i=1, \cdots, n)$,

$a$ and $d$ for all $x$ in $N \cap \hat{S}_{y}$,

(b) $\quad \sum_{i, j=1}^{n-1} a_{i j}(x, u, 0) D_{i j} \tau(x) \geqq-B(\tau(x))$ for all $x$ in $N \cap \hat{S}_{y}$,

(c) $\quad \liminf _{\substack{x \rightarrow y \\ x \in \hat{S}_{\mathrm{v}}}} \frac{\sum_{i=1}^{n} b_{i}(x, u, 0) D_{i} \tau(x)}{B(\tau(x))}>-\infty$, and

(d) $\quad \lim _{\substack{x \rightarrow y \\ x \in \hat{s}_{y}}} \frac{d(x, u, 0) \tau(x)}{B(\tau(x))}>-\infty$.

We shall see in Theorems $2.1-2.3$ that the part of condition $Q(a)$ relating to the function $a$ can be weakened to

$$
a(x, u, D u)-a(x, u, 0) \leqq f(\tau(x),|D u(x)|) .
$$

In analogous theorems concerning negative minima, the inequality used is

$$
a(x, u, D u)-a(x, u, 0) \geqq-f(\tau(x),|D u(x)|) .
$$

The basis of this paper is the following extension of Theorem 3.1 of Dow and Výborný (1972). For brevity we call a vector $l$ admissible at a point $y$ in $\partial G$ if it emanates from $y$ into $\hat{S}_{y}$ at an angle less than $\pi / 2$ with the inner normal to $\partial G$ at $y$.

THEOREM 1.1. Let $y \in \partial G$ with $S_{y} \neq \varnothing$, let $u \in C\left(\bar{S}_{y}\right) \cap D^{2}\left(\hat{S}_{y}\right)$, and let $|D u|$ and $\left|D_{i j} u\right|(i, j=1, \cdots, n-1)$, be bounded by the constants $L$ and $K$ in $\hat{S}_{y}$ in 
some open neighbourhood $N$ of $y$. Let $P$ satisfy condition $Q$ at $y$ relative to $u$ in $N$ and also the non-degeneracy condition relative to $u$

$$
\lim _{\substack{x \rightarrow y \\ x \in \hat{S}_{y}}} \inf _{i, j=1}^{n-1} a_{i j}(x, u, 0) D_{i} \tau(x) D_{j} \tau(x)>\beta>0 .
$$

Let the constant $c$ associated with $f$ satisfy

$$
c>c_{0} \frac{M}{\beta} \equiv\left((n-1)^{2} K+n L+U+1\right) \frac{M}{\beta}
$$

where $U=u(y)$. Suppose also $P u \geqq 0, b_{n}(x, u, 0) \leqq 0, a(x, u, 0) \leqq 0$ and $d(x, u, 0)$ $\leqq 0$ on $N \cap \hat{S}_{y}$. Lastly, let $u(y) \geqq 0$ and $u(x)<u(y)=U$ for all $x$ in $\bar{S}_{y} \cap \bar{N}$ except $y$. Then

$$
\limsup _{\substack{x \rightarrow y \\ x \in l}} \frac{u(x)-u(y)}{|x-y|}<0
$$

for every admissible vector $l$ at $y$.

The proof is a simple modification of that of Theorem 3.1 of Dow and Výborný (1972). The auxiliary function $w(x)=u(x)+z(\tau(x))$ is now defined on $\bar{N} \cap \bar{S}_{y}$ instead of $\overline{N \cap G}$ and

with

$$
z(t)=\frac{1}{c_{1}} \int_{0}^{\tau} \phi(t) \exp \left(\frac{3}{\beta} \int_{0}^{t} B(s) d s\right) d t
$$

$$
c_{1}=M \exp \left(\frac{3}{\beta} \int_{0}^{A_{1}} B(t) d t\right) .
$$

One shows by contradiction that $w$ cannot attain its maximum over $\bar{S}_{y} \cap \bar{N}$ at a point of $N \cap \hat{S}_{y}$. The auxiliary linear operator $E_{0}$ is replaced by

$$
P_{0} w(x)=\sum_{i, j=1}^{n-1} a_{i j}(x, u, 0) D_{i j} w(x)+\sum_{i=1}^{n} b_{i}(x, u, 0) D_{i} w(x)+d(x, u, 0) w(x) .
$$

A close examination of the proof will show that the function $\tau$ need only be defined for points in $\hat{S}_{y}$, that is, on the smaller sets $\bar{N}_{y}$ where $N_{y}=\bar{N} \cap \hat{S}_{y}$.

\section{Maximum principles and uniqueness theorems for single operators}

Subscripts " $H y$ " or " $H$ ' will indicate dependence on a particular point $y$ in the boundary of a domain $H$ or just on the particular domain $H$ itself. For a sphere $H$ with $\bar{H} \subset G$ and a point $y$ in $\bar{H}$, let $S_{y}(H)$ denote the propagation set relative to the domain $H$. The notation $\hat{S}_{y}(H)$ means the closure of $S_{y}(H)$ relative to $H$. Obviously if $Y \in \bar{G}$ and $y \in \hat{S}_{Y} \cap \bar{H}$ then $S_{y}(H) \subset \hat{S}_{Y}$. 
If $Y \in G$, if $u \in D^{2}\left(\hat{S}_{Y}\right)$ and if each $D_{i j} u$ is bounded on compact subsets of $S_{Y}$, the operator $P$ is said to satisfy the interior condition $I C$ on $G$ relative to $u$ and $Y$ if, for every open sphere $H$ whose closure lies in $G$ and every point $y$ in $\partial H \cap S_{Y}$ not at the "top" or "bottom" of $H$ relative to the $x_{n}$-coordinate, we have

(i) $P$ satisfies condition $Q$ on the domain $H$ at $y$ relative to $u$,

(ii) $\liminf _{\substack{x \rightarrow y \\ x \in \hat{S}_{y(H)}}} \sum_{i, j=1}^{n-1} a_{i j}(x, u, 0) D_{i} \tau_{H y}(x) D_{j} \tau_{H y}(x)>\beta_{H y}>0$,

(iii) each constant $c_{H y}$ associated with the function $f_{H y}$ satisfies

$$
c_{H y}>\left((n-1)^{2} K_{H y}+n L_{H y}+U_{H y}+1\right) \frac{M_{H y}}{\beta_{H y}}
$$

where $K_{H y}, L_{H y}$ and $U_{H y}$ are bounds for $D_{i j} u, D u$ and $u$ over $S_{y}(H)$.

THEOREM 2.1. Let $Y \in G$, let $u \in D^{2}\left(\hat{S}_{Y}\right)$ and let $D_{i j} u$ be bounded on compact subsets of $S_{Y}$. Let $P u \geqq 0, b_{n}(x, u, 0) \leqq 0, a(x, u, 0) \leqq 0$ and $d(x, u, 0) \leqq 0$ on $\hat{S}_{Y}$. Suppose $P$ satisfies condition IC on $G$ relative to $u$ and $Y$. Under these conditions, if $u$ attains a non-negative maximum over $\hat{S}_{Y}$ ay $Y$ then $u$ is constant on $T_{Y}$.

Actually the condition $a(x, u, 0) \leqq 0$ need only hold in a neighbourhood of each maximum of $u$.

The proof follows Nirenberg's. (See (1953), Theorem 1.) He proved first that if a sphere $H$ whose closure lies in $G$ contains no maximum points of $u$ in its interior but has one such point in its boundary, then that point is either the "top" or "bottom" of $H$ relative to the $x_{n}$-coordinate. If the point is a maximum relative to $S_{Y}$ and lies in $S_{Y}$, then this conclusion follows in our case from Theorem 1.1. The rest of the proof is the same as Nirenberg's.

To show that maxima spread to the rest of $S_{Y}$, we must strengthen condition $I C$. For each open sphere $H$ whose closure lies in $G$, the functions $\tau$ and $f$ must depend only on $H$ and not vary with individual points of the boundary. Also, $B$ must be constant and depend only on $H$, and $f$ must satisfy additional conditions.

The operator $P$ is said to satisfy condition $Q I$ relative to $u$ and $Y$ on a sphere $H$ in $G$ if

(1) there is a function $f$ satisfying conditions (i), (ii) and (iii) listed in $\$ 1$ and (iv) $f(t, \sigma \phi) \leqq \sigma f(t, \phi)$ for all $\sigma>0$, (v) for every $\varepsilon$ with $0<\varepsilon<A$, there is $\xi>0$ such that $f$ is bounded over $[\varepsilon, A] \times[0, \xi]$;

(2) there is a function $\tau$ defined on $\bar{H} \cap S_{Y}$ satisfying

(i) $\tau \in C^{1}\left(\bar{H} \cap S_{Y}\right) \cap D^{2}\left(H \cap \hat{S}_{Y}\right)$,

(ii) $\tau=0$ on $\partial H \cap S_{Y}$ and $\tau>0$ on $H \cap \hat{S}_{Y}$,

(iii) $|D \tau|<M_{H}<\infty$ on $H \cap \hat{S}_{Y}$, and $|D \tau|>0$ on $\partial H \cap \hat{S}_{Y}$, except possibly at the "top" and "bottom" of $H$, 
(iv) $\tau(x)<A$ on $H \cap \hat{S}_{Y}$ where $A$ is the constant associated with $f$ in conditions (1) (iii) and (v) above;

(3) $f$ and $\tau$ satisfy condition $Q(a)$ over $H \cap \hat{S}_{Y}$ and there is a constant $B_{H}>0$ such that $-B_{H}$ bounds

$$
b_{i}(x, u, 0), d(x, u, 0) \text { and } \sum_{i, j=1}^{n-1} a_{i j}(x, u, 0) D_{i j} \tau(x) \text { below }
$$

Next, if $Y \in G, u \in D^{2}\left(\hat{S}_{Y}\right)$ and if $D_{i j} u$ is bounded on compact subsets of $\hat{S}_{Y}$, we say $P$ satisfies the interior condition $P I C$ on $G$ relative to $u$ and $Y$ if, for every open sphere $H$ whose closure lies in $G$,

(i) $P$ satisfies condition $Q I$ relative to $u$ and $Y$ on $H$,

(ii) there is $\beta_{H}>0$ such that

$$
\liminf _{\substack{x \rightarrow y \\ x \in H \cap \hat{S}_{Y}}} \sum_{i, j=1}^{n-1} a_{i j}(x, u, 0) D_{i} \tau_{H}(x) D_{j} \tau_{H}(x)>\beta_{H}>0
$$

for all points $y$ in $\partial H \cap S_{Y}$ except perhaps those at the "top" and "bottom" of $H$, (iii) the constant $c_{H}$ associated with $f_{H}$ satisfies

$$
c_{H}>c_{0} \frac{M_{H}}{\beta_{H}} \exp \left(\frac{A B_{H}\left(1+n M_{H}+A\right)}{\beta_{H}}\right)
$$

where $c_{0}=\left((n-1)^{2} K_{H y}+n L_{H y}+U_{H y}+1\right)$ and $K_{H y}, L_{H y}$ and $U_{H y}$ are bounds for $D_{i j} u, D u$ and $u$ over $\bar{S}_{y}(H)$.

THEOREM 2.2. Suppose the hypothesis of Theorem 2.1 holds with condition $I C$ replaced by $P I C$ and with $b_{n}(x, u, 0)$ bounded away from zero by a negative constant on compact subsets of $G$. Then $u$ is constant on $S_{Y}$.

Proof. We combine the method used in Theorem 1.1 (see Dow and Výborný (1972)) with a method used by Il'in, Kalashnikov and Oleinik (1962). Proceed by contradiction. Assume $u$ is not constant on $\hat{S}_{Y}$. There is $x^{\prime}$ in $S_{Y}$ such that $u\left(x^{\prime}\right)<u(Y) ; x^{\prime}$ is connected to $Y$ by a path $\Gamma$ along which $x_{n}$ is non-decreasing from $x^{\prime}$ to $Y$. Follow $\Gamma$ from $x^{\prime}$ towards $Y$ to the first point $x^{0}$ where $u\left(x^{0}\right)=u(Y)$. There is an open spherical neighbourhood $H$ of $x^{0}$ with $\bar{H} \subset G$. Notice that $S_{Y} \cap \bar{H}=\left\{x \in \bar{H}: x_{n} \leqq x_{n}^{0}\right\}$. Choose a point $x^{\prime \prime} \neq x^{0}$ in $\Gamma \cap H$. Obviously $x_{n}^{\prime \prime}<x_{n}^{0}$ because of Theorem 2.1. Let $C$ denote the cylindrical set

$$
C=\left\{x \in H: x_{n}^{\prime \prime}<x_{n}<x_{n}^{0}\right\} .
$$

Obviously $\vec{C} \subset S_{Y}$. Let $\Gamma_{1}=\left\{x \in \bar{C}: x_{n}=x_{n}^{\prime \prime}\right\}, \quad \Gamma_{2}=\bar{C} \cap \partial H$ and $\Gamma_{3}=\partial C$ $-\left(\Gamma_{1} \cap \Gamma_{2}\right)$. Since $u<u\left(x^{0}\right)$ on $\Gamma$ between $x^{\prime \prime}$ and $x^{0}$, Theorem 2.1 implies $u \equiv u\left(x^{0}\right)$ on $\bar{\Gamma}_{3}$ and $u<u\left(x^{0}\right)$ on the rest of $\bar{C}$. The function $u$ is bounded away from $u(Y)$ on $\Gamma_{1}$; that is, there is a constant $m_{1}$ such that $u(x)<m_{1}<u\left(x^{0}\right)$ 

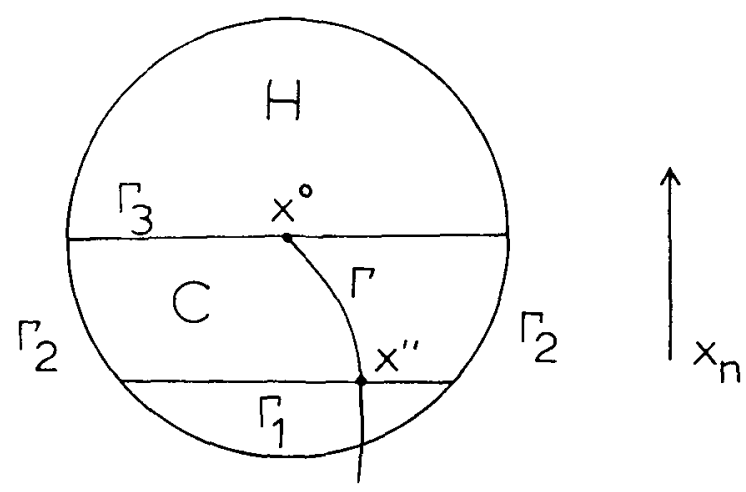

$=u(Y)$ for all $x$ in $\Gamma_{1}$. Also, condition PIC (ii) implies there are positive constants $\zeta$ and $\beta_{H}$ such that

$$
\sum_{i, j=1}^{n-1} a_{i j}(x, u(x), 0) D_{i} \tau(x) D_{j} \tau(x)>\beta_{H}>0
$$

on the set $Z_{1}=\left\{x \in C \cap \Gamma_{3}\right.$ : dist $\left.\left(x, \Gamma_{2}\right)<\zeta\right\}$. Let $a_{0}$ be the infimum of $\tau$ over the set $Z_{2}=\left(C \cup \Gamma_{3}\right)-Z_{1}$. The continuity of $\tau$ implies $a_{0}>0$. The conditions on $f$ guarantee there is a positive constant $\xi$ such that $f$ is bounded on $\left[a_{0}, A\right] \times[0, \xi]$ Further, there is a supersolution $\phi$ to the problem $\phi^{\prime}=c f(t, \phi)$ on $[0, A]$ with $0<\phi<\xi$.

Define the auxiliary function $w$ on $\bar{C}$ by $w(x)=u(x)+\sigma(x)$ where

$$
\sigma(x)=\left(u(Y)-m_{1}\right) z(\tau(x)) \exp \left(-k_{0}\left(x_{n}-x_{n}^{\prime \prime}\right)\right) .
$$

The constant $k_{0}$ will be chosen later and $z$ is the function defined by

$$
z(\tau)=\frac{1}{c_{1}} \int_{0}^{\tau} \phi(t) e(t) d t
$$

where for brevity

$$
e(t)=\exp \left(\frac{B_{H}\left(1+n M_{H}+A\right) t}{\beta_{H}}\right) \text { and } c_{1}=M_{H} e(A) .
$$

Clearly $\phi$ can be taken small enough that $z(\tau)<1$ on $[0, A]$.

On $\Gamma_{1}, w(x) \leqq m_{1}+\left(u(Y)-m_{1}\right)=u(Y)$; on $\Gamma_{2}, z=0$ so that $w(x) \leqq u(Y)$; and on $\Gamma_{3}, w(x)=u(Y)+\sigma(x)>u(Y)$. Since $u(Y) \geqq 0$, it follows that $w$ attains a positive maximum over $\bar{C}$ somewhere in $C \cup \Gamma_{3}$, say at a point $y$. We show that this is impossible. Define the operator $P_{0}$, linear relative to $w$, by

$$
P_{0} w(x)=\sum_{i, j=1}^{n-1} a_{i j}(x, u, 0) D_{i j} w(x)+\sum_{i=1}^{n} b_{i}(x, u, 0) D_{i} w(x)+d(x, u, 0) w(x) .
$$


Obviously $P_{0} w(y) \leqq 0$ because $b_{n}(x, u, 0) \leqq 0, d(x, u, 0) \leqq 0$, and $w$ has a positive maximum at $y$. On the other hand, $P_{0} w(y)>0$ by the following argument.

$$
\begin{aligned}
P_{0} w(y) \geqq & P_{0} w(y)-P u(y) \geqq-c_{0} f(\tau(y),|D u(y)|) \\
& +\sum_{i, j=1}^{n-1} a_{i j}(y, u, 0) D_{i j} \sigma(y)+\sum_{i=1}^{n} b_{i}(y, u, 0) D_{i} \sigma(y)+d(y, u, 0) \sigma(y) \\
\geqq & -c_{0} f(\tau(y),|D u(y)|) \\
& +\left(u(Y)-m_{1}\right) e^{-k_{0}\left(y_{n}-x_{n}{ }^{\prime \prime}\right)}\left\{z^{\prime \prime}(\tau) \sum_{i, j=1}^{n-1} a_{i j}(y, u, 0) D_{i} \tau D_{j} \tau\right. \\
& +z^{\prime}(\tau)\left[\sum_{i, j=1}^{n-1} a_{i j}(y, u, 0) D_{i j} \tau+\sum_{i=1}^{n} b_{i}(y, u, 0) D_{i} \tau\right] \\
& \left.+z(\tau)\left(-k_{0} b_{n}(y, u, 0)+d(y, u, 0)\right)\right\} .
\end{aligned}
$$

Let $-\beta_{1}$ be the supremum of $b_{n}(x, u, 0)$ over $\bar{H} \cap \bar{S}_{Y}$; by hypothesis $\beta_{1}>0$. Now at the point $y$

$$
\begin{aligned}
|D u| & =|D \sigma|=\left(u(Y)-m_{1}\right) e^{-k_{0}\left(y_{1}-x_{n}{ }^{\prime \prime}\right.} z^{\prime}(\tau(y))|D \tau(y)| \\
& <\left(u(Y)-m_{1}\right) e^{-k_{0}\left(y_{n}-x_{n}{ }^{\prime \prime}\right)} \phi(\tau(y)) .
\end{aligned}
$$

Thus $f(\tau,|D u|) \leqq f\left(\tau,\left(u(Y)-m_{1}\right)\right) e^{-k_{0}\left(y_{n}-x_{n}{ }^{\prime \prime}\right)} \phi(\tau(y))$ and by condition $Q I$ 1(iv) on $f$

$$
\left.f(\tau,|D u|) \leqq\left(u(Y)-m_{1}\right) e^{-k_{0}\left(y_{n}-x_{n}{ }^{n}\right.}\right) f(\tau, \phi(\tau(y))) .
$$

Therefore,

$$
\begin{aligned}
& P_{0} w(y) \geqq\left(u(Y)-m_{1}\right) e^{-k_{0}\left(y_{n}-x_{n}{ }^{\prime \prime}\right)}\left\{-c_{0} f(\tau, \phi(\tau(y)))\right. \\
&\left.\quad+z^{\prime \prime}(\tau) \sum_{i, j=1}^{n-1} a_{i j}(y, u, 0) D_{i} \tau D_{j} \tau-z^{\prime}(\tau)\left(B_{H}+n B_{H} M_{H}\right)+z(\tau)\left(k_{0} \beta_{1}-B_{H}\right)\right\} .
\end{aligned}
$$

Now

$$
\begin{aligned}
-z^{\prime}(\tau) & \left(B_{H}+n B_{H} M_{H}\right)-B_{H} z(\tau) \\
& =-\frac{1}{c_{1}} \phi(\tau) e(\tau) B_{H}\left(1+n M_{H}\right)-\frac{B_{H}}{c_{1}} \int_{0}^{\tau} \phi(t) e(t) d t \\
& \geqq-\frac{1}{c_{1}}\left[\phi(\tau) e(\tau) B_{H}\left(1+n M_{H}\right)+B_{H} A \phi(\tau) e(\tau)\right] \\
& =-\frac{1}{c_{1}} \phi(\tau) e(\tau) B_{H}^{*}
\end{aligned}
$$

where $B_{H}^{*}=B_{H}\left(1+n M_{H}+A\right)$. Therefore, 


$$
\begin{aligned}
P_{0} w(y) \geqq & \left(u(Y)-m_{1}\right) e^{-k_{0}\left(y_{n}-x_{,}{ }^{\prime \prime}\right)}\left[-c_{0} f(\tau, \phi(\tau(y)))\right) \\
& \left.+z^{\prime \prime}(\tau) \sum_{i, j=1}^{n-1} a_{i j}(y, u, 0) D_{i} \tau D_{j} \tau-\frac{1}{c_{1}} \phi(\tau) e(\tau) B_{H}^{*}+k_{0} \beta_{1} z(\tau)\right] .
\end{aligned}
$$

Denote the expression in square brackets by $J$. The remainder of the proof is devoted to showing that $k_{0}$ can be chosen large enough that $J>0$. We treat the cases $y \in Z_{1}$ and $y \in Z_{2}$ separately. If $y \in Z_{2}$, then

$$
J \geqq-c_{0} f(\tau(y), \phi(\tau(y)))-\frac{1}{c_{1}} \phi(A) e(A) B_{H}^{*}+k_{0} \beta_{1} z(\tau)
$$

because $z^{\prime \prime}>0$ and $P$ is degenerate elliptic-parabolic. Now $f$ is bounded below over $Z_{2}$ because for $x$ in $Z_{2}$ we have $(\tau(x), \phi(\tau(x))) \in\left[a_{0}, A\right] \times[0, \xi]$. Also $z(\tau)$ is bounded away from zero on $Z_{2}$. Therefore, if $k_{0}$ is large enough, $J>0$ for $y$ anywhere in $Z_{2}$. Fix $k_{0}$ at this particular value. Go back to inequality $\left({ }^{*}\right)$. For $y$ in $Z_{1}$,

$$
\begin{aligned}
J & >-c_{0} f(\tau, \phi(\tau))+\beta_{H} z^{\prime \prime}(\tau)-\frac{1}{c_{1}} e(\tau) \phi(\tau) B_{H}^{*} \\
& =-c_{0} f(\tau, \phi(\tau))+\frac{\beta_{H}}{c_{1}} \phi^{\prime}(\tau) \\
& =\frac{\beta_{H}}{c_{1}}\left[\phi^{\prime}(\tau)-\frac{c_{0} c_{1}}{\beta_{H}} f(\tau, \phi(\tau))\right] \geqq \frac{\beta_{H}}{c_{1}}\left[\phi^{\prime}(\tau)-c_{H} f(\tau, \phi(\tau))\right] \geqq 0 .
\end{aligned}
$$

Therefore, $P_{0} w(y)>0$. This proves the theorem.

To deduce the usual boundary maximum principle of Hopf type, one must first remove from Theorem 1.1 the condition $u(x)<u(y)$ at points of the boundary by deforming the boundary near $y$ as in Dow and Výborný (1972). This requires strengthening other parts of the hypothesis.

The operator $P$ satisfies condition $Q B$ relative to $u$ at a point $y$ in $\partial G$ if $P$ satisfies condition $Q$ with (b) and (c) replaced by

$\left(\mathrm{b}^{\prime}\right)\left|D_{i j} \tau(x)\right| \leqq B(\tau(x))$ on $\bar{G} \cap \bar{N}$ for $i, j=1, \cdots, n-1$ and $a_{i j}(x, u(x), 0)$ is continuous at $y$ for $i, j=1, \cdots, n-1$, and

$\left(\mathrm{c}^{\prime}\right)\left|b_{i}(x, u, 0)\right| \leqq B(\tau(x))$ on $N \cap S_{y}$, and if, in addition,

(i) $f$ is a non-increasing function of its first variable $t$,

(ii) $\tau$ can be extended to a continuously differentiable function on $N$,

(iii) the function $B$ is non-increasing.

If $y \in \partial G$, if $u \in C\left(\bar{S}_{y}\right) \cap D^{2}\left(\hat{S}_{y}\right)$ and if $D u$ and $D_{i j} u$ are bounded on $\hat{S}_{y}$ (at least in a neighbourhood of $y$ ) then $P$ satisfies the boundary condition $B C$ relative to $u$ at $y$ if 
(i) $P$ satisfies condition $Q B$ relative to $u$ at $y$,

(ii) $\sum_{i, j=1}^{n-1} a_{i j}(y, u(y), 0) D_{i} \tau(y) D_{j} \tau(y)>\beta>0$, and

(iii) the constant $c$ associated with $f$ satisfies

$$
c>\left((n-1)^{2} K+n L+U+1\right) \frac{M}{\beta}
$$

where $K, L$ and $U$ are bounds for $D_{i j} u, D u$ and $u$ over $\hat{S}_{y} \cap N$.

We shall assume that the boundary of $G$ is so well behaved that if $y \in \partial G$ then

$$
\hat{S}_{y} \subset\left(\cup S_{y n}\right)^{\wedge}
$$

for any sequence of points $y^{n}$ in $S_{y}$ converging to $y$.

Theorem 2.3. Let $y \in \partial G$ and $S_{y} \neq \varnothing$. Let $u \in C\left(\bar{S}_{y}\right) \cap D^{2}\left(\hat{S}_{y}\right)$ and let $D u$ and $D_{i j} u$ be bounded on $\hat{S}_{y}$ for $i, j=1, \cdots, n-1$. Let $P u \geqq 0, a(x, u, 0) \leqq 0$, $d(x, u, 0) \leqq 0$ and let $b_{n}(x, u, 0)$ be negative and bounded away from zero on compact subsets of G. Suppose P satisfies condition PIC on $G$ relative to $u$ and $x$ for every $x$ in $\hat{S}_{y}$ and every sphere in $G$ (at least in a neighbourhood of $y$ ) and condition $B C$ relative to $u$ at $y$. Then $u$ cannot attain a non-negative maximum over $\hat{S}_{y}$ at $y$ unless either $u$ is constant on $\hat{S}_{y}$ or

$$
\limsup _{\substack{x \rightarrow y \\ x \in l}} \frac{u(x)-u(y)}{|x-y|}<0
$$

for every admissible vector $l$ at $y$.

Proof. Suppose $u$ attains a non-negative maximum over $S_{y}$ at $y$. Either there is a neighbourhood $N_{1}$ of $y$ such that $u(x)<u(y)$ for all $x$ in $N_{1} \cap S_{y}$ or there is no such neighbourhood. Suppose the latter: there is a sequence of points $y^{n}$ in $\hat{S}_{y}$ converging to $y$ and $u\left(y^{n}\right)=u(y)$. Since $\hat{S}_{y^{n}} \subset \hat{S}_{y}$, the function $u$ attains a nonnegative maximum over $\hat{S}_{y n}$ at $y^{n}$. By Theorem $2.2, u \equiv u(y)$ on $\hat{S}_{y^{n}}$. Because we assumed $\hat{S}_{y} \subset\left(\cup S_{y^{n}}\right)^{\wedge}$, this implies $u \equiv u(y)$ on $\hat{S}_{y}$.

Suppose on the other hand there is a neighbourhood $N_{1}$ of $y$ such that $u(x)$ $<u(y)$ for all $x$ in $N_{1} \cap \hat{S}_{y}$. It is an easy matter to modify Theorem 6.1 of [4] to provide the result

$$
\limsup _{\substack{x \rightarrow y \\ x \in l}} \frac{u(x)-u(y)}{|x-y|}<0 .
$$

In this case the deformation is made in a direction $x_{j}$ with $j \neq n$. To ensure $\tau_{1}$ is well-defined, a neighbourhood $N$ in $N_{1}$ is used which is a cylinder with axis in the $x_{j}$ direction. The theorem follows from this.

To simplify matters the consequent uniqueness theorem will be given for a domain of the form $G=G_{1} \times(0, X)$ where $G_{1}$ is a bounded, open, connected 
subset of $R^{n-1}$ and $X>0$. Label the parts of the boundary: $Z=\bar{G}_{1} \times\{0\}$, $\Sigma=\partial G \cap\left(0<x_{n}<X\right)$, and $\gamma=\partial G-(Z \cap \Sigma)$. We define the parabolic mixed boundary value problem $P^{*}$ as follows.

A function $u$ is a solution to the problem $P^{*}$ if $u \in C^{1}(\bar{G}) \cap D^{2}(G), P u=0$ on $G, B u(x) \equiv \alpha(x) D_{l} u(x)+\beta(x) u(x)=0$ on $\Sigma$, and $u(x)=0$ on $Z$. (Actually one does not need $u \in C^{1}$ on $Z$ or $\gamma$.) The functions $\alpha$ and $\beta$ are defined on $\Sigma$ and satisfy the inequalities $\alpha \geqq 0, \beta \leqq 0$ and $\alpha^{2}+\beta^{2}>0 ; l$ denotes any admissible vector at $x$ and may vary with $x$.

THEOREM 2.4. Let $u$ be a solution to the problem $P^{*}$ and let $D_{i j} u$ be bounded. Let $a(x, u, 0) \cdot u(x) \leqq 0$ and $d(x, u, 0) \leqq 0$ on $G$. Let $b_{n}(x, u, 0)$ be negative and bounded away from zero on compact subsets of $G$. Suppose $P$ satisfies condition $P I C$ on $G$ relative to $u$ and $x$ for every $x$ in $G$ and every sphere in $G$. Suppose $P$ also satisfies condition $B C$ relative to $u$ and $y$ for every point $y$ in $\Sigma$. Then $u \equiv 0$ on $G$.

(Actually the condition $a(x, u, 0) \cdot u(x) \leqq 0$ need only hold in a neighbourhood of each maximum and minimum of $u$.)

The proof is standard and follows from Theorems 2.2 and 2.3 and their analogues for negative minima. (See, for example, Výborný (1958), Theorem 4.)

One can combine Theorem 2.4 with a technique used by Kusano (1963) to derive conditions under which solutions to boundary value problems of the form

$$
\begin{gathered}
\text { " } P u=0 \text { on } G, \\
B u(x) \equiv \alpha(x) D_{l} u(x)+\beta(x) u(x)=g(x) \text { on } \Sigma, \\
u(x)=\delta(x) \text { on } Z,
\end{gathered}
$$

with $\alpha \geqq 0, \beta \leqq 0$ and $\alpha^{2}+\beta^{2}>0$ " are unique or have at most a constant difference. For such a result, one assume the coefficients $a_{i j}, b_{i}$ and $d$ are functions of $x$ and $D u$ alone. Also one needs strengthened versions of conditions PIC and $B C$. For example, inequalities of the type $Q(a)$ must be replaced by

$$
|e(x, p+D u)-e(x, D u)| \leqq f(\tau(x),|p|) .
$$

Other inequalities must be replaced in a similar manner.

\section{Generalization to systems}

Consider the system of inequalities

$$
\left(W^{+}\right) P_{\mu} u_{\mu}(x)+\sum_{v=1}^{p} h_{\mu \nu}\left(x, u_{\mu}(x), D u_{\mu}(x)\right) u_{\nu}(x) \geqq 0, \mu=1, \cdots, p,
$$

where the $P_{\mu}$ are degenerate elliptic-parabolic operators relative to $u_{\mu}$ of the type described in $\S 1$. The coefficients of $P_{\mu}$ and $h_{\mu \nu}$ are defined on $\bar{G} \times R^{1} \times R^{n-1}$. Dependence on $\mu$ is denoted by sub- or superscripts $\mu$ : for example, $a_{i j}^{\mu}\left(x, u_{\mu}, D u_{\mu}\right)$. 
The coupling coefficients $h_{\mu v}$ are assumed to satisfy $h_{\mu \nu} \geqq 0$ for $\mu \neq v$ and $\sum_{v=1}^{p} h_{\mu v}$ $\leqq 0$ for $\mu=1, \cdots, p$.

Observe that if $U$ is a non-negative constant and $u_{v} \leqq U$ for all $v=1, \cdots, p$, then

$$
P_{\mu} u_{\mu}+h_{\mu \mu}\left(u_{\mu}-U\right) \geqq P_{\mu} u_{\mu}+\sum_{\nu=1}^{p} h_{\mu v}\left(u_{v}-U\right) \geqq P_{\mu} u_{\mu}+\sum_{v=1}^{p} h_{\mu \nu} u_{\nu} \geqq 0
$$

for every $\mu$. Thus if $u$ satisfies the weakly coupled system $W^{+}$then $u$ satisfies the system of separate inequalities

$$
P_{\mu} u_{\mu}+h_{\mu \mu}\left(u_{\mu}-U\right) \geqq 0, \mu=1, \cdots, p .
$$

Similarly, if $V$ is a non-positive constant and $u_{v} \geqq V$ for all $v=1, \cdots, p$, then the weakly coupled system

$$
\left(W^{-}\right) P_{\mu} u_{\mu}+\sum_{v=1}^{p} h_{\mu \nu} u_{v} \leqq 0, \mu=1, \cdots, p
$$

implies the system of separate inequalities

$$
P_{\mu} u_{\mu}+h_{\mu \mu}\left(u_{\mu}-V\right) \leqq 0, \mu=1, \cdots, p .
$$

Thus to prove a maximum or a minimum principle for a system it is sufficient to prove it for a single inequality of the form $P u+h(u-U) \geqq 0$ with appropriate conditions on $h$. Notice that this is not a trivial extension of the case $P u \geqq 0$ in that the terms $h(u-U)$ and $h(u-V)$ cannot be absorbed into the terms $d(x, u, D u) u$ and $a(x, u, D u)$ of $P$. For example, the maximum principles require $a(x, u, 0) \leqq 0$. From the inequalities satisfied by $h_{\mu \nu}$ it follows that $h_{\mu \mu} \leqq 0$ for $\mu=1, \cdots, p$. Thus $h_{\mu \mu}\left(u_{\mu}-U\right) \geqq 0$.

Theorem 1.1 generalizes as follows:

THEOREM 3.1. Let $y \in \partial G$ and let $u=\left(u_{1}, \cdots, u_{p}\right)$ satisfy the system $W^{+}$. Let $u_{\mu}$ be one of the components of $u$. Suppose the hypothesis of Theorem 1.1 holds relative to $P_{\mu}$ and $u_{\mu}$ and, in addition, $h_{\mu \mu}$ satisfies the conditions

$$
\begin{gathered}
Q(e)\left|h_{\mu \mu}\left(x, u_{\mu}(x), 0\right)-h_{\mu \mu}\left(x, u_{\mu}(x), D u_{\mu}(x)\right)\right| \leqq f\left(\tau(x),\left|D u_{\mu}(x)\right|\right) \text { on } N \cap \hat{S}_{y}, \text { and } \\
Q(f) \liminf _{\substack{x \rightarrow y \\
x \in \hat{S}_{v}}} \frac{h_{\mu \mu}\left(x, u_{\mu}(x), 0\right) \tau(x)}{B(\tau(x))}>-\infty .
\end{gathered}
$$

Finally, suppose $u_{\mu}(y)>u_{\mu}(x)$ for all $x$ in $N \cap S_{y}$ except $y, u_{\mu}(y) \geqq u_{v}(x)$ for all $x$ in $N \cap \bar{S}_{y}$ and all $\nu \neq \mu$, and $u_{\mu}(y) \geqq 0$. Then

$$
\lim \sup _{\substack{x \rightarrow y \\ x \in l}} \frac{u_{\mu}(x)-u_{\mu}(y)}{|x-y|}<0
$$

for every admissible vector $l$ at $y$. 
Proof. Let $U=u_{\mu}(y)$. As pointed out above, the conclusion holds if Theorem 1.1 holds for operators that include a term of the form $h(x, u, D u)(u-U)$ with $h$ satisfying $Q(e)$ and $Q(f)$. This is easily shown. In the proof of Theorem 1.1 given in Dow and Výborný (1972) replace

$$
\exp \left(\frac{1}{\beta} \int_{0}^{t} B(s) d s\right) \text { by } \exp \left(\frac{4}{\beta} \int_{0}^{t} B(s) d s\right)
$$

in the definition of $z$ and similarly in the constant $c_{1}$. Let the auxiliary linear operator be

$$
\begin{aligned}
P_{0} w= & \sum_{i, j=1}^{n-1} a_{i j}(x, u, 0) D_{i j} w+\sum_{i=1}^{n} b_{i}(x, u, 0) D_{i} w \\
& +d(x, u, 0) w(x)+h(x, u, 0)[w(x)-U] .
\end{aligned}
$$

Since $h(x, u, 0) \leqq 0$ and $w\left(x_{0}\right) \geqq u(y)=U \geqq 0$ at a maximum $x_{0}$ of $w$, we still have $P_{0} w\left(x_{0}\right) \leqq 0$. The contradictory result $P_{0} w\left(x_{0}\right)>0$ follows as before using the conditions assumed on $h_{\mu \mu}$.

Theorems 2.1-2.3 extend to systems in the same way. In Theorem 2.1, inequalities $Q(e)$ and $Q(f)$ are added to condition $Q$ of $I C$. The conclusion is that if $u_{\mu}$ attains a non-negative maximum over $\hat{S}_{Y}$ at $Y$ and $u_{v} \leqq u_{\mu}(Y)$ on $S_{Y}$ then $u_{\mu}$ is constant on $T_{Y}$. In Theorem 2.2, $Q(e)$ is added to condition $Q I(3)$ of $P I C$, $h_{\mu \mu}(x, u, 0)$ is assumed bounded below on compact subsets of $G$ and in $P I C$ (iii) the constants $c_{H}$ must satisfy

$$
c_{H}>c_{0} \frac{M_{H}}{\beta_{H}} \exp \left(\frac{A B_{H}\left(1+n M_{H}+2 A\right)}{\beta_{H}}\right) .
$$

In Theorem 2.3, the same changes are made to condition $P I C$ as for Theorem 2.2 and inequalities $Q(e)$ and $Q(f)$ are added to condition $Q B$. We also assume $u_{v}(x) \leqq u_{u}(Y)$ on $\hat{S}_{Y}$.

From these theorems and their analogues for minima we can prove a uniqueness theorem. A function $u=\left(u_{1}, \cdots, u_{p}\right)$ is a solution to the problem $(W)$ if $u_{\mu} \in C^{1}(\bar{G}) \cap D^{2}(G)$ and

$$
\begin{aligned}
P_{\mu} u_{\mu}+\sum_{\nu=1}^{p} h_{\mu v} u_{v} & =0 \text { on } G, \\
B_{\mu} u_{\mu} & =\alpha_{\mu} D_{l} u_{\mu}+\beta_{\mu} u_{\mu}=0 \text { on } \Sigma, \text { and } \\
u_{\mu}(x) & =0 \text { on } Z
\end{aligned}
$$

for $\mu=1, \cdots, p$ with $\alpha_{\mu} \geqq 0, \beta_{\mu} \leqq 0$ and $\alpha_{\mu}^{2}+\beta_{\mu}^{2}>0 ; l$ is any admissible vector at $x$ and may vary with $x$ and $\mu$.

THEOREM 3.2. Let $u$ be a solution to the boundary value problem $W$ with $D_{i j}$ bounded. Let $a^{\mu}\left(x, u_{\mu}, 0\right) \cdot u_{\mu}(x) \leqq 0$ and $d^{\mu}\left(x, u_{\mu}, 0\right) \leqq 0$ on $G$ for $\mu=1, \cdots, p$. 
Let each $b_{n}^{\mu}\left(x, u_{\mu}, 0\right)$ be negative and bounded away from zero on compact subsets of $G$. Suppose each $P_{\mu}$ satisfies condition PIC on $G$ relative to $u_{\mu}$ and $y$ for every point $y$ in $G$ and condition $B C$ relative to $u_{\mu}$ and $y$ for every point $y$ in $\Sigma$ with changes made to PIC and BC as for Theorems 2.2 and 2.3. Suppose also that $\alpha_{\mu}=0$ at some point of $\Sigma$ for each $\mu$. Then $u \equiv 0$ on $G$.

(As before, the condition $a^{\mu}\left(x, u_{\mu}, 0\right) \cdot u_{\mu}(x) \leqq 0$ need only hold in a neighbourhood of each maximum and minimum.)

Proof. Suppose the conclusion is false. Then $u_{\mu}(x) \neq 0$ for some $\mu$ and some $x$ in $G$. Suppose $u_{\mu}(x)>0$. Let $U=\max \left\{u_{v}(x): x \in G\right.$ and $\left.v=1, \cdots, p\right\}$. It follows that $U>0$ and that $U$ is attained by one of the functions $u_{r}$ at some point of $S_{x}$. Since $u_{v} \leqq U$ for all $v$, Theorems 2.2 and 2.3 and their analogues for minima hold and the rest of the proof follows in the standard way.

\section{A more general parabolic operator}

Fix a function $u$ in $D^{2}(G)$. For $i=1, \cdots, n$ and $k=1, \cdots, n-1$ let $\alpha_{i k}$ be a real-valued function defined on some open set $\Omega$ containing $\bar{G}$. We assume $\alpha_{i k}$ is Lip 1 and differentiable on $\Omega$ although for interior maximum principles $\alpha_{i k}$ need only be Lip 1 and differentiable on $G$. Since $u$ is fixed, $\alpha_{i k}$ may be permitted to depend on $u$ as well as $x$. For $w$ in $D^{2}(G)$ let $D_{k} w=\sum_{i=1}^{n} \alpha_{i k} D_{i} w$ for $k=1, \cdots, n-1$ and $\bar{D} w=\left(\bar{D}_{1} w, \cdots, \bar{D}_{n-1} w\right)$. Denote by $\bar{P}$ the operator

$$
\begin{aligned}
\bar{P} u(x)= & \sum_{k, l=1}^{n-1} a_{k l}(x, u, \bar{D} u) \bar{D}_{k} \bar{D}_{l} u+\sum_{i=1}^{n} b_{i}(x, u, \bar{D} u) D_{i} u \\
& +d(x, u, \bar{D} u) u+a(x, u, \bar{D} u) .
\end{aligned}
$$

This is similar to operator introduced by Redheffer (1971). It differs in that nonlinearity of the first order term appears here in the coefficients of $D_{i} u$, and a term $d(x, u, \bar{D} u) u$ is included in order to generalize Pucci's result (1958); also, we have limited the summation of $k$ and $l$ to $1, \cdots, n-1$. The functions $a, d, b_{i}$ and $a_{k l}$ are defined on $G \times R^{1} \times R^{n-1}$. We assume the $b_{i}(x, u, 0)$ are defined and Lip 1 on $\Omega$ although again this is not always needed.

The operator $\bar{P}$ is assumed to be degenerate elliptic-parabolic in the sense that

$$
\sum_{k, l=1}^{n-1} a_{k l}(x, u, 0) \lambda_{k} \lambda_{l} \geqq 0
$$

for all $\lambda$ in $R^{n-1}$ and all $x$ in $G$. This is somewhat different from the corresponding assumption made by Redheffer (1971).

With Hill (1970) we define a trajectory of a vector field $Z$ to be a curve $\Gamma$ in $R_{n}$ satisfying the parametric condition $x^{\prime}(t)=Z(x(t))$ and $x^{\prime}(t) \neq 0$. Then a diffusion trajectory is a trajectory generated by one of the vector fields 


$$
X_{k}(x)=\left(\alpha_{1 k}(x), \cdots, \alpha_{n k}(x)\right), k=1, \cdots, n-1 .
$$

A drift trajectory is a trajectory generated by the vector field

$$
X_{0}(x)=\left(b_{1}(x, u, 0), \cdots, b_{n}(x, u, 0)\right) ;
$$

it is given the orientation induced by $X_{0}$. A generalized trajectory is a trajectory generated by a linear combination $Z=\sum_{k=0}^{n-1} \gamma_{k} X_{k}$ with $\gamma_{k}$ constant, $\gamma_{0} \geqq 0$ and with orientation induced by $Z$. If $\gamma_{0}=0$ such a trajectory is called a generalized diffusion trajectory.

Points $x$ and $y$ are connected by a chain of trajectories oriented from $y$ to $x$ if there is finite sequence of points $y=P_{1}, \cdots, P_{r}=x$ such that each consecutive pair $P_{i}$ and $P_{i+1}$ belong to the same diffusion, drift, generalized diffusion or generalized trajectory oriented from $P_{i}$ to $P_{i+1}$ in the appropriate cases. If $H$ is an open subset of $G$ and $y \in \bar{H}$, the propagation set $S_{y}(H)$ is the set of points $x$ in $H$ connected to $y$ by a chain of trajectories all lying in $H$ except possibly for $y$ and oriented from $y$ to $x$. For $y$ in $\vec{H}, T_{y}$ is the set of points in $H$ connected to $y$ by a chain of generalized diffusion trajectories all lying in $H$ except possibly for $y$. When $H=G$, we simply write $S_{y}$ and $T_{y}$. Closure of $S_{y}$ relative to $G$ (or $H$ ) is denoted by $S_{y}$ (or $S_{y}(H)$ ) and relative to $R^{n}$ by $\bar{S}_{y}$ (or $\bar{S}_{y}(H)$ ); similarly for $T_{y}$.

Obviously $T_{y} \subset S_{y}$. Also, if $x \in T_{y}$ then $T_{x} \subset T_{y}$ and if $x \in \hat{T}_{y}$ then $\hat{T}_{x} \subset \hat{T}_{y}$. Similarly, if $x \in S_{y}$ then $S_{x} \subset S_{y}$, and if $x \in \hat{S}_{y}$ then $\hat{S}_{x} \subset \hat{S}_{y}$. Hill proved these facts for $y$ in $G$ and his method applies equally well for $y$ in $\partial G$ (see Hill (1970), p. 216). As Redheffer pointed out, a theorem of Bony (1969) implies that all points of $S_{y}$ are connected to $y$ by a chain consisting only of diffusion and drift trajectories and oriented from $y$ to $x$. Let $S_{y}^{N}$ and $T_{y}^{N}$ denote the propagation sets described in $\$ 1$ and denoted there by $S_{y}$ and $T_{y}$. It is not difficult to prove the following proposition, which gives conditions under which $S_{y}$ and $T_{y}$ as defined above reduce to $S_{y}^{N}$ and $T_{y}^{N}$.

Proposition. Let $y \in G$. If $X_{1}, \cdots, X_{n-1}$ span $x_{1} \cdots x_{n-1}$-space for all $x$ in $G$ then $\hat{T}_{y}=\hat{T}_{y}^{N}$. If also $b_{n}(x, u, 0)<0$ on $G$, then $\hat{S}_{y}=\hat{S}_{y}^{N}$.

Let $y \in \partial G$. If $X_{1}, \cdots, X_{n-1}$ span $x_{1} \cdots x_{n-1}$-space for all $x$ in $G \cap\{y\}$, then the following hold.

(a) If $G \cap\left(x_{n}=y_{n}\right)$ contains an interior cone at $y$ then $\hat{T}_{y}$ is the connected component of $G \cap\left(x_{n}=y_{n}\right)$ containing the cone and having $y$ in its boundary.

(b) Let $b_{n}(x, u, 0)<\beta_{n}<0$ on $G$. Suppose there is a neighbourhood of $y$ such that any two points $x^{0}$ and $x^{1}$ in $\bar{N} \cap G$ with $x_{n}^{0} \leqq x_{n}^{1}$ can be connected by a path in $N \cap G$ with $x_{n}$ non-decreasing from $x^{0}$ to $x^{1}$. Suppose also that $G$ has an open interior cone at $y$ that has non-void intersection with the half-space $\left(x_{n} \leqq y_{n}\right)$. Then $\hat{S}_{y}=\hat{S}_{y^{*}}^{N}$

(A statement analogous to (b) holds when $b_{n}(x, u, 0)$ is positive.) 
The theorems of $\S \S 1-3$ are easily extended to this operator. We assume throughout that $X_{1}, \cdots, X_{n-1}$ span $x_{1} \cdots x_{n-1}$-space for all $x$ in $G$. In condition $Q$, $D u$ is replaced by $\bar{D} u$ in (a) and $D_{i j}$ and $a_{i j}$ by $\bar{D}_{k} \bar{D}_{l}$ and $a_{k l}$ in (b). Theorem 1.1 holds in this setting if $K$ is an upper bound for $\left|\bar{D}_{k} \bar{D}_{l} u\right|$, if $D_{i}, D_{j}$ and $a_{i j}$ are replaced by $\bar{D}_{k}, \bar{D}_{l}$ and $a_{k l}$ in the non-degeneracy condition, and if the constant $M$ in the inequality involving $c$ is replaced by

$$
T=\sup \{|\bar{D} \tau(x)|: x \in \bar{N} \cap G\} .
$$

This last quantity is finite because $\alpha_{i k}$ and $D \tau$ are bounded. We call this Theorem 1.1*. The proof follows as before except that $\bar{P}_{0} w \leqq 0$ is derived from the fact that at an interior maximum

$$
\begin{aligned}
\bar{D}_{k} w(x) & =0, \\
\sum_{k, l=1}^{n-1} \lambda_{k} \lambda_{l} \bar{D}_{k} \bar{D}_{l} w(x) & \leqq 0 \text { for all } \lambda \in R^{n-1}, \text { and } \\
X_{0}(x) \cdot D w(x) & =\sum_{i=1}^{n-1} b_{i}(x, u, 0) D_{i} w(x) \leqq 0 .
\end{aligned}
$$

This is proved as in Lemma 4 of Redheffer (1971).

A generalization of Theorem 2.1 , namely Theorem $2.1^{*}$, holds with analogous changes in condition $I C$ and the hypothesis. (Furthermore, positive maxima spread to $T_{y}$ for more general operators in which summation in the second order term runs from 1 to some $m$ with $1 \leqq m<n$.) A theorem of Bony (see Bony (1969) and Redheffer (1971)), Lemma 3) implies the following.

LEMMA 4.1. If $y \in G$ and all spheres $H$ in $G$ for which $u<u(y)$ in $H \cap S_{y}$ but $u\left(x^{0}\right)=u(y)$ for some point $x^{0}$ in $\partial H \cap \hat{S}_{y}$ have the property that the unit normal $v$ to $H$ at $x^{0}$ is orthogonal to each of the vectors $X_{k}\left(x^{0}\right), k=1, \cdots, n-1$, then $u \equiv u(y)$ on any generalized diffusion trajectory passing through $y$.

Similarly, if each such sphere $H$ has the property that $v \cdot X_{0}\left(x^{0}\right) \leqq 0$, then $u \equiv u(y)$ on any drift trajectory passing through $y$.

If each such sphere has both properties, another theorem due to Bony (Bony (1969) and Redheffer (1971)), Lemma 2) implies $u \equiv u(Y)$ on $S_{y}$.

Thus to prove Theorem $2.1^{*}$ it is sufficient to show that all such spheres have the property $X_{k}\left(x^{0}\right) \cdot v=0$ for $k=1, \cdots, n-1$. Reduce the size of $H$ if necessary so that $y$ is the only point of $\partial H \cap S_{y}$ where $u=u(y)$. Suppose $X_{k}\left(x^{0}\right) \cdot v \neq 0$ for some $k$. Since $X_{1}\left(x^{0}\right), \cdots, X_{n-1}\left(x^{0}\right)$ span $x_{1} \cdots x_{n-1}$-space, this implies $x^{0}$ is not the "top" or "bottom" of $H$ relative to the $x_{n}$-coordinate. Therefore, Theorem $1.1^{*}$ applied to the domain $H$ at $x^{0}$ implies

$$
\tilde{D}_{k} u\left(x^{0}\right)=X_{k}\left(x^{0}\right) \cdot D u\left(x^{0}\right)= \pm \lim _{\substack{x \rightarrow y \\ x \in \Gamma}} \frac{u(x)-u(y)}{|x-y|} \neq 0,
$$


where $\Gamma=\Gamma_{k} \cap H$ and $\Gamma_{k}$ is the diffusion trajectory corresponding to $X_{k}$ and passing through $x^{0}$. However, $x^{0}$ is a local maximum of $u$ relative to $\hat{S}_{y}$. Thus $\bar{D}_{k} u\left(x_{0}\right)=0$. This contradiction proves Theorem $2.1^{*}$.

A Theorem 2.2* with analogous modifications in conditions $P I C$ can be proved from Lemma 4.1 by showing that all such spheres $H$ have the property that $X_{0}\left(x^{0}\right) \cdot v \leqq 0$. Suppose on the contrary $v \cdot X_{0}\left(x^{0}\right)>0$. We have already proved $X_{1}\left(x^{0}\right), \cdots, X_{n-1}\left(x^{0}\right)$ are orthogonal to $v$ at $x^{0}$. Since they span $x_{1} \cdots x_{n-1}$ space, $v$ must be parallel to the $x_{n}$-axis. Now $b_{n}\left(x^{0}, u, 0\right)<0$ and $v \cdot X_{0}\left(x^{0}\right)>0$ which implies that $v$ points in the negative $x_{n}$-direction. So $x_{n}<x_{n}^{0}$ for all $x$ in $H$. The proof now proceeds as for Theorem 2.2 with the path $\Gamma$ replaced by the integral curve $\Gamma_{0}$ of $X_{0}$ passing through $x^{0}$. Obviously $\Gamma_{0}$ points into $H$.

Theorem 2.3 and 2.4 have similar analogues and all of these can be generalized to systems as in $\$ 3$.

\section{Acknowledgements}

This paper comprises part of a thesis [Dow (1972)] presented to the University of Queensland in fulfillment of the requirements for the degree of Doctor of Philosophy. The work was carried out under a University of Queensland Research Scholarship. I am grateful to Professor R. Výborný for his supervision and advice during the study and for reading this manuscript.

\section{References}

K. Ako (1968), 'Semi-decomposable systems and vector subfunctions', Funkcialaj Ekvacioj 8, 91-97.

J.-M. Bony (1969), 'Principe du maximum, inegalité de Harnack et unicité du problème de Cauchy pour les opérateurs elliptiques dégénérés', Ann. Inst. Fourier (Grenoble) 19, fasc. 1, 277-304.

M. A. Dow (1972), Maximum principles for some quasilinear degenerate elliptic-parabolic operators of second order, Dissertation, (University of Queensland, 1972).

M. A. Dow and R. Výborný (1972), 'Maximum principles for some quasilinear second order partial differential equations', Rend. Sem. Mat. Univ. Padova 47, 331-351.

C. D. Hill (1970), 'A sharp maximum principle', Indiana Univ. Math. J. 20, 213-229.

O. Horáček and R. Výborný (1966), 'Über eine fastlineare partielle Differentialgleichung vom nichthyperbolischen Typus', Comment. Math. Univ. Carolinae 7, 3, 261-264.

A. M. Il'in, A.C. Kalashnikov and O. A. Oleinik (1962), 'Linear second order equations of parabolic type', (Russian) Uspekhi Mat. Nauk 17, 1-146. English transl. Russian Math. Surveys 17, 1-143.

N. V. Khusnytdinova (1967), 'The limiting moisture profile during infiltration into a homogeneous soil', (Russian) Prikl. Mat. Mekh. 31, 770-776. English transl. J. Appl. Math. Mech. 31, 783-789.

T. Kusano (1963), 'On the maximum principle for quasilinear parabolic equations of the second order', Proc. Japan Acad. 39, 211-216.

O. A. Ladyzhenskaia, V. A. Solonnikova and N. N. Ural'tseva (1968), Linear and quasilinear 
equations of parabolic type, Translations of Mathematical Monographs 23 (Amer. Math. Soc., 1968).

P. P. Moisak (1971), 'A generalized maximum principle and an estimate of the error in Rothé's method for second order parabolic systems', (Ukrainian) Dopovidi Akad. Nauk Ukrain. RSR Ser A, 109-113, 187.

L. Nirenberg (1953), 'A strong maximum principle for parabolic equations', Comm. Pure Appl. Math. 6, 167-177.

M. H. Protter and H. F. Weinberger (1967), Maximum principles in differential equations (Prentice Hall, 1967).

C. Pucci (1957-58), 'Properietá di massimo e minimo delle soluzioni di equazioni a derivate parziali del secondo ordine di tipo ellittico e parabolico I, II', Atti Accad. Naz. Lincei Rend. Cl. Sci. Fis. Mat. Natur. (8) 23, 370-375; 24, 3-6.

R. M. Redheffer (1962), 'An extension of certain maximum principles', Monatsh. Math. 66, 32-42.

R. M. Redheffer (1971), 'A sharp maximum principle for nonlinear inequalities', Indiana Univ. Math. J. 21, 227-248.

D. W. Stroock (1970), 'On certain systems of parabolic equations', Comm. Pure Appl. Math. 23, 447-457.

R. Výborný (1958), 'Some basic properties of solutions to boundary value problems for parabolic partial differential equations', (Russian) Czechoslovak Math. J. 8 (83), 537-551.

R. Výborný (1963), 'On certain extension of the maximum principle', Differential Equations and Their Applications (Proc. Conf. Prague, 1962) pp. 223-228. (Publ. House Czechoslovak Acad. Sci., Prague; Academic Press, New York, 1963).

J. Wasowski(1970), 'Maximum principles for a certain strongly elliptic system of linear equations of second order', Bull. Acad. Polon. Sci. Sér. Sci. Math. Astronom. Phys. 18, 741-745.

Department of External Studies

University of Queensland, Brisbane

Australia 